\title{
No seven year itch for Metabolomics
}

\section{Royston Goodacre}

Published online: 27 December 2011

(C) Springer Science+Business Media, LLC 2011

Welcome to the Eighth Volume of Metabolomics!

It continues to be an exciting journey for the journal and I hope you are as pleased as I to see that after seven publication years that the impact of the journal remains very healthy. This is of course due to the great papers, reviews and opinion articles that we publish and I thank these Authors very much for choosing Metabolomics as the home for their exciting and interesting work. It goes without saying that a very big thank you also goes to the many Reviewers and Editorial Board who spend some of their valuable time assessing these articles and doing such a good job. Unfortunately as some of you know we now reject just over half of the papers that are submitted for review and we expect this rejection rate to increase over the coming years.

Again we have a nice front cover from Drs Rick Beger and Thomas Colatsky highlighting the biomarker qualification process at the US FDA. It is appropriate that the journal features some of the most important work on the front of the journal and I hope you find these informative.

This year we are increasing our publication frequency from four to six issues per annum, and this is a reflection of the fact that last year the number of manuscripts submitted to the journal has more than doubled; importantly without a drop in scientific quality.

When appropriate Metabolomics publishes themed or what we refer to as Special Issues and these are edited by Guest Editors. In 2011 we are publishing two of these: the first Special Issue covers Data Analysis for Metabolomics and is edited by Drs Johan Westerhuis and Jeroen Jansen; the second is edited by Drs Rick Dunn and Thomas Hankemeier and the theme is Mass Spectrometry for Metabolomics. I am very grateful to all these Guests Editors for their hard work and commitment to delivering a great line up of primary papers, reviews and tutorials. If any of you have thoughts on future special issues I would be very interested to hear about them, as I would be to hear about potential review articles or tutorials. I can be contacted at the coordinates below.

I remind you that Metabolomics also has a twitter presence (http://twitter.com/metabolomics) and there are regular updates on the Journal tweeted there.

I hope as ever that you enjoy this issue and continue to read and send your best work to Metabolomics.

I wish you all the very best for a successful and peaceful 2012.

R. Goodacre $(\square)$

School of Chemistry and Manchester Interdisciplinary

Biocentre, University of Manchester, 131 Princess Street,

Manchester M1 7DN, UK

e-mail: Roy.Goodacre@manchester.ac.uk 